\title{
Substructure in bulk velocities of Milky Way disk stars
}

\author{
Jeffrey L. Carlin ${ }^{1,2}$, James DeLaunay ${ }^{1,2}$, Heidi Jo Newberg ${ }^{2}$, Licai Deng ${ }^{3}$, Daniel Gole ${ }^{2,4}$, \\ Kathleen Grabowski ${ }^{2}$, Ge $\mathrm{Jin}^{5}$, Chao $\mathrm{Liu}^{3}$, Xiaowei Liu ${ }^{6}$, A-Li Luo ${ }^{3}$, Haibo Yuan ${ }^{6}$, Haotong \\ Zhang $^{3}$, Gang Zhao ${ }^{3}$, Yongheng Zhao ${ }^{3}$
}

\begin{abstract}
We find that Galactic disk stars near the anticenter exhibit velocity asymmetries in both the Galactocentric radial and vertical components across the mid-plane as well as azimuthally. These findings are based on LAMOST spectroscopic velocities for a sample of $\sim$ 400, 000 F-type stars, combined with proper motions from the PPMXL catalog for which we have derived corrections to the zero points based in part on spectroscopically discovered galaxies and QSOs from LAMOST. In the region within $2 \mathrm{kpc}$ outside the Sun's radius and $\pm 2 \mathrm{kpc}$ from the Galactic midplane, we show that stars above the plane exhibit net outward radial motions with downward vertical velocities, while stars below the plane have roughly the opposite behavior. We discuss this in the context of other recent findings, and conclude that we are likely seeing the signature of vertical disturbances to the disk due to an external perturbation.
\end{abstract}

Subject headings: Galaxy: disk — Galaxy: structure — Galaxy: kinematics and dynamics - Galaxy: stellar content — stars: kinematics and dynamics

\section{Introduction}

A variety of instabilities can produce non-axisymmetric features near the midplane of the Galaxy, including resonances due to the bar and/or spiral arms (e.g., Fux 2001; Quillen \& Minchev 2005; - Antoja et al. 2009; Minchev et al. 2009, 2010; Quillen et al. 2011), and (possibly associated) processes such as radial migration (e.g., Sellwood \& Binney 2002; Havwood 2008; Minchev \& Famaev

\footnotetext{
${ }^{1}$ Equal first authors.

${ }^{2}$ Department of Physics, Applied Physics and Astronomy, Rensselaer Polytechnic Institute, Troy, NY 12180, USA, carlij@rpi.edu

${ }^{3}$ Key Lab for Optical Astronomy, National Astronomical Observatories, Chinese Academy of Sciences, Beijing 100012, China

${ }^{4}$ Department of Physics, SUNY-Geneseo

${ }^{5}$ University of Science and Technology of China, Hefei 230026, China

${ }^{6}$ Kavli Institute for Astronomy and Astrophysics, Peking University, Beijing 100871, China; Department of Astronomy, Peking University, Beijing 100871, China
} 
2010). In contrast, any vertical structures that are seen must have been excited by an external means such as a passing satellite galaxy or some other halo substructure. Vertical density and velocity structures have been shown to arise in model Milky Way disks due to perturbation by a Sagittarius (Sgr) sized dwarf galaxy (Gómez et al. 2013, see also Purcell et al. 2011). Widrow et al. (2012) showed the existence of vertical, wavelike structures in stellar density from Sloan Digital Sky Survey (SDSS) data. Stars in this structure apparently also exhibit vertical motions (perpendicular to the disk), suggesting that the structures are coherent perturbations in the disk. Widrow et al. (2012) modeled the effect of a massive perturber on the vertical structure of the Galactic disk, and showed that fairly short-lived vertical waves are produced in such encounters. Analytically, these authors showed that vertical perturbations should have wavelengths slightly less than $2 \mathrm{kpc}$. Gómez et al. (2012) showed that peaks in the energies of SDSS-selected thick disk stars are consistent with their predictions of merger-induced waves in the disk, which can be long-lived if the perturber is sufficiently massive.

Velocity structure in both the vertical and radial directions was also seen in a sample of RAVE (RAdial Velocity Experiment; Steinmetz et al. 2006) stars within $2 \mathrm{kpc}$ of the Sun by Williams et al. (2013). This study probed mostly the fourth Galactic quadrant, and thus contains the majority of its data inside the solar radius. Nonetheless, apparently similar wavelike structures are seen in the different volumes probed by the RAVE (Williams et al. 2013) and SDSS (Widrow et al. 2012) studies.

In this Letter, we extend the work of these previous studies using a much larger, independent data set. We use spectra from the LAMOST survey combined with corrected proper motions from the PPMXL catalog to derive three-dimensional space velocities for a large sample of stars with known spectral types. From our sample of $\sim 400,000$ F-type stars near the Galactic anticenter, we demonstrate the existence of a kinematic asymmetry above and below the Galactic plane in the $2 \mathrm{kpc}$ cylindrical shell just outside of the Sun's radius. The asymmetry is present in both the radial and vertical components of the Galactocentric velocities.

\section{Data}

\subsection{LAMOST spectra}

As of June 2013, the LAMOST (Large Sky Area Multi-Object Fiber Spectroscopic Telescope: Cui et al. 2012; Zhao et al. 2012) survey has completed its first season of official operations, in addition to a year of pilot survey data collection. In these first two observing seasons, $\sim 1.7$ million spectra have been obtained that have sufficient signal to noise $(S / N)$ for velocity/redshift measurements. The majority of these stars were observed as part of the Milky Way structure portion of the survey, known as LEGUE (LAMOST Experiment for Galactic Understanding and Exploration; Deng et al. 2012). LAMOST observes at a resolution of $R \equiv \frac{\Delta \lambda}{\lambda} \sim 1800$ over a wavelength range of $3800 \lesssim \lambda \lesssim 9100 \AA$, which produces spectra similar to those from SDSS (Yanny et al. 2009). 
Table 1: Selection criteria for the stellar sample. Ranges in $V_{R}, V_{\theta}$, and $V_{Z}$ were chosen to exclude high-velocity, nearby halo stars.

\begin{tabular}{c}
\hline classified as F-type star \\
$S / N>5$ in $g, r$, and $i$ \\
error in $V_{\text {helio }}<10 \mathrm{~km} \mathrm{~s}^{-1}$ \\
error in $\mu_{\alpha} \cos \delta, \mu_{\delta}<10 \mathrm{mas} \mathrm{yr}^{-1}$ \\
$-150<V_{R}<150 \mathrm{~km} \mathrm{~s}^{-1}$ \\
$-400<V_{\theta}<-100 \mathrm{~km} \mathrm{~s}^{-1}$ \\
$-150<V_{Z}<150 \mathrm{~km} \mathrm{~s}^{-1}$ \\
$7.8<R_{\mathrm{GC}}<9.8 \mathrm{kpc}$ \\
$-2.0<Z_{\mathrm{GC}}<2.0 \mathrm{kpc}$ \\
\hline
\end{tabular}

From the $\sim 1.7$ million spectra available to the collaboration, $\sim 1.3$ million are stellar spectra with $S / N>5$ in the SDSS $g, r$, and $i$ bands. Although the process for determination of stellar parameters (i.e., $\log g, T_{\text {eff }}$, and $[\mathrm{Fe} / \mathrm{H}]$ ) from LAMOST spectra is still being refined, the radial velocities (RVs) and spectral types are well measured (see, e.g., Luo et al. 2012). The spectra are mostly of bright $(r \lesssim 17)$ stars spanning a large range of photometric colors (for more information about LEGUE pilot survey target selection, see Carlin et al. 2012; Chen et al. 2012; Yang et al. 2012; Zhang et al. 2012, and for a description of the LAMOST Galactic anticenter survey and the Xuyi photometric survey from which its targets were selected, see Liu et al. 2013). Such a sample will inevitably contain a majority of nearby main sequence stars, since these dominate the number density along any line of sight.

For this study, we selected stars classified as F-type by the LAMOST pipeline that have $J, H$, and $K_{S}$ magnitudes from the Two Micron All Sky Survey (2MASS; Skrutskie et al. 2006). Magnitudes were corrected for extinction using $\mathrm{E}(\mathrm{B}-\mathrm{V})$ derived from the maps of Schlegel et al. (1998), with coefficients for 2MASS bands from Cardelli et al. (1989). Due to site conditions and the location of the Guoshoujing telescope, most of the LEGUE data are concentrated near the Galactic anticenter. The $S / N$, velocity error, spatial, and velocity criteria used to select the sample are given in Table 1. These criteria yield 380,159 LAMOST F-stars, and an additional $\sim 10,737$ RAVE stars (see Section 2.4).

We derive distances based on calibration to Hipparcos (Perryman et al. 1997) data with spectroscopic information from Gontcharov (2006). We select F-type stars from the Gontcharov catalog and remove giants and subdwarfs. For stars with reliable parallaxes, we derive average absolute magnitudes of $M_{K_{S}}=1.57,1.83$, and 2.18 for F2, F5, and F9 stars, respectively (using \pm 1 sub-class; e.g., "F2" includes F1-F3 stars). Distances to LAMOST F-stars were derived using the absolute magnitude of the type nearest the observed LAMOST spectral sub-type. Note that because we used $K_{S}$-band magnitudes, the effects on our derived distances of overestimating extinction to nearby, 
Table 2: Proper motion fits as a function of RA, Dec.

\begin{tabular}{ccccccc}
\hline fit & $\mathrm{a}$ & $\mathrm{b}$ & $\mathrm{c}$ & $\mathrm{d}$ & $\mathrm{e}$ & $\mathrm{f}$ \\
\hline$\mu_{\alpha} \cos \delta$ & 1.8986 & $-4.1637 \mathrm{E}-02$ & $1.0376 \mathrm{E}-04$ & $1.2363 \mathrm{E}-04$ & $-5.1269 \mathrm{E}-02$ & $4.0594 \mathrm{E}-04$ \\
$\mu_{\delta}$ & -3.2247 & $1.0490 \mathrm{E}-02$ & $-2.9027 \mathrm{E}-05$ & $2.1413 \mathrm{E}-04$ & $-6.3930 \mathrm{E}-02$ & $1.3776 \mathrm{E}-03$
\end{tabular}

$\mu_{\mathrm{fit}}=a+(b \alpha)+\left(c \alpha^{2}\right)+(d \alpha \delta)+(e \delta)+\left(f \delta^{2}\right)$, where $\mu_{\mathrm{fit}}$ is the proper motion being fit, in mas $\mathrm{yr}^{-1}$, and $(\alpha, \delta)$ are right ascension and declination, both in degrees.

low-latitude stars are minimal.

\subsection{Proper motion corrections}

We match the LAMOST data to PPMXL (Roeser et al. 2010). PPMXL proper motions of QSOs are known to contain systematic shifts that vary with position on the sky (see, e.g., Wu et al. 2011). We derive new corrections to the proper motion reference frame using 100,919 QSOs from the catalog of Véron-Cetty \& Véron (2010) plus 12,619 QSOs and galaxies spectroscopically identified by LAMOST (and also in PPMXL). After removing objects with large proper motion errors, we fit polynomials in two dimensions to the mean proper motions of QSOs (which should be zero) as a function of position on the sky. Coefficients for these fits are given in Table 2. Our fits show similar behavior to that seen in $\mathrm{Wu}$ et al. (2011) - the $\mu_{\alpha} \cos \delta$ residuals are low (i.e., negative) in the north

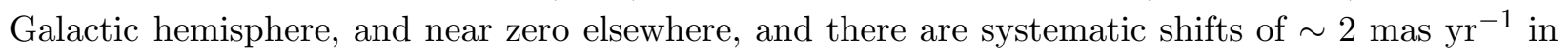
both proper motion dimensions. The $\mu_{\delta}$ fit increases mostly as a function of declination. In all subsequent discussion, the proper motions were pre-corrected with these polynomial fits before using the data for analysis.

\subsection{Coordinate system and velocity calculations}

We derive positions and velocities in Galactic Cartesian $\left(X Y Z_{\mathrm{GC}}\right)$ and cylindrical $\left(R_{\mathrm{GC}}, \theta, Z_{\mathrm{GC}}\right)$ coordinate systems with origins at the Galactic center. The Cartesian $X_{\mathrm{GC}}$-axis is positive toward the Galactic center, with the Sun at $X_{\mathrm{GC}}=-7.8 \mathrm{kpc}$ (McMillan \& Binney 2010). $Y_{\mathrm{GC}}$ is in the direction of Galactic rotation, and $Z_{\mathrm{GC}}$ is positive toward the north Galactic pole. The distribution of the LAMOST sample in $X Y Z_{\mathrm{GC}}$ is shown in Figure 1. Galactocentric cylindrical coordinates place the Sun at $(R, \theta, Z)_{\mathrm{GC}}=\left(7.8 \mathrm{kpc}, 0^{\circ}, 0 \mathrm{kpc}\right)$, with $\theta$ increasing in the same direction as Galactic longitude. Three-dimensional Cartesian $(U, V, W)$ velocities are corrected to a Galactocentric frame by removing the Schönrich et al. (2010) values for the solar peculiar motion, $(U, V, W)_{\odot}=(11.1,12.24,7.25) \mathrm{km} \mathrm{s}^{-1}$, and a local standard of rest velocity of $247 \mathrm{~km} \mathrm{~s}^{-1}$ 

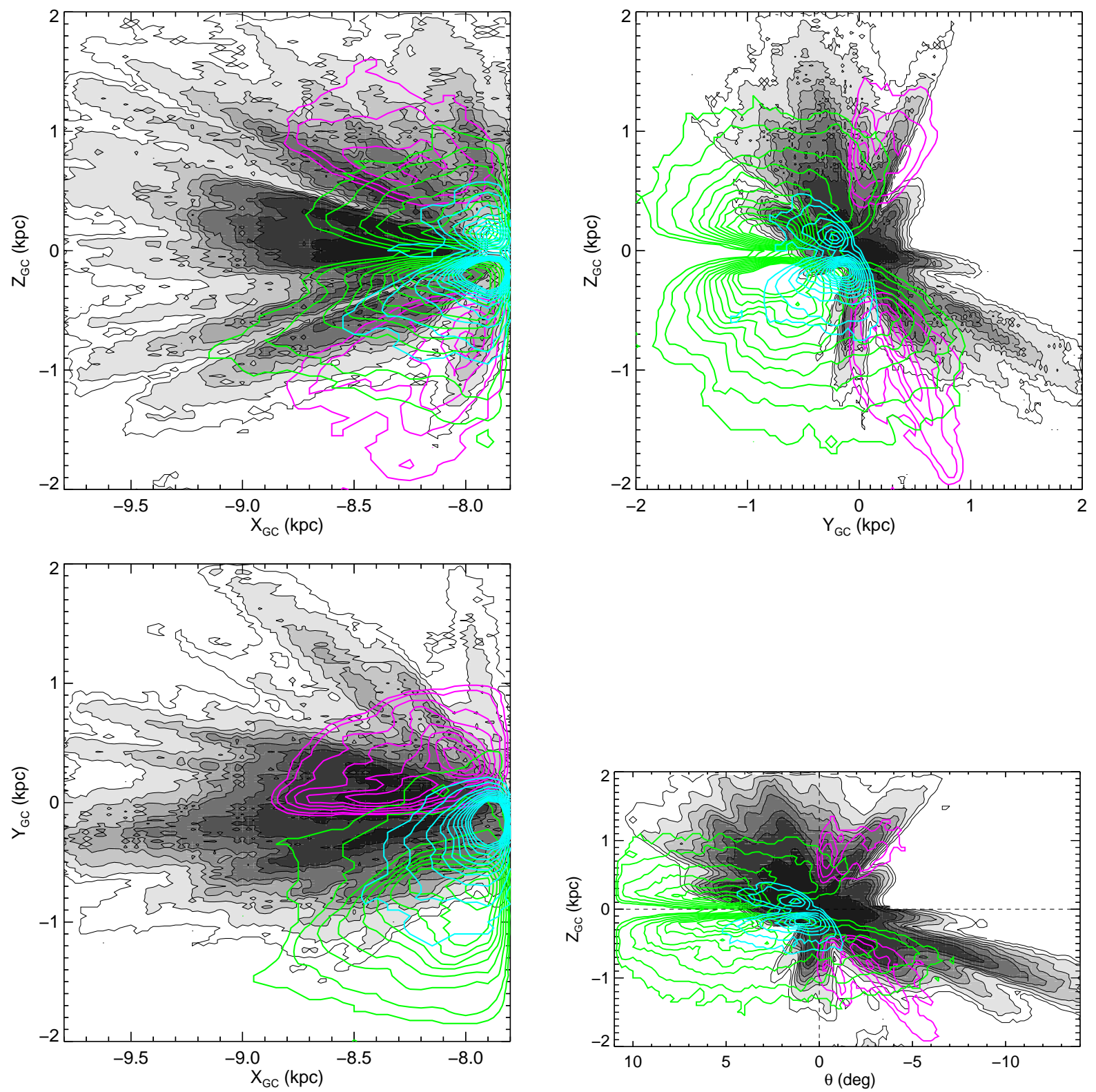

Fig. 1.- Spatial distribution, in Galactic coordinates, of the stars with LAMOST spectra used in this study. The Sun is at $(X, Y, Z)_{\mathrm{GC}}=(-7.8,0,0) \mathrm{kpc}$. Smoothed, grayscale contours represent the number of stars in $250 \times 250$ pc bins, with levels of $5,10,20,30,40,50,75,100$, and 200 stars per bin. Magenta contours show the SDSS data from Widrow et al. (2012), green contours the RAVE red clump sample from Williams et al. (2013), and cyan contours denote the RAVE sample used in our study.

(McMillan \& Binney 2010). Because $V_{\theta}$ increases in the direction of Galactic longitude (opposite 
the direction of rotation), the cylindrical rotation velocity in the solar neighborhood is $-247 \mathrm{~km} \mathrm{~s}^{-1}$.

\subsection{RAVE spectra}

To more completely sample the sky in the volume of the Galaxy we are studying, we added data from the southern-hemisphere RAVE survey Data Release 3 (Siebert et al. 2011) to our sample. Using distances given by the RAVE catalog (from Zwitter et al. 2010), we select stars from the same volume of the Milky Way as the LAMOST sample (see Table 1 for selection criteria). This adds 10,737 stars, most of which are in a small volume of the third Galactic quadrant just below the midplane. The addition of the publicly available RAVE data acts as confirmation of the LAMOST results in the third quadrant.

\section{Velocity asymmetries among nearby disk stars}

We explore velocity structure within the cylindrical shell bounded by $7.8<R_{\mathrm{GC}}<9.8 \mathrm{kpc}$ and $-2.0<Z<2.0 \mathrm{kpc}$ as a function of position. Figure 2 displays the mean $V_{R}$ (upper panels) and $V_{Z}$ (lower panels) velocities of stars binned spatially. The plots in the left column are centered on the Galactic anticenter $\left(Z=0 \mathrm{kpc}, \theta=0^{\circ}\right)$, with the third Galactic quadrant to the left side, and the second quadrant to the right. Plots on the right display the velocity dependences with Galactocentric radius. We display the data as distinct dots so that it is obvious where LAMOST has observed, and which areas lack coverage. Small filled squares in Figure 2 represent the mean values for RAVE stars discussed in Section 2.4. These data were selected with the same spatial criteria and error cuts as those from LAMOST. The RAVE data provide a small but important amount of additional coverage in $Z$ and $\theta$ that is not present in the LAMOST data.

If all of the relatively nearby stars in our sample were in disk-like orbits, one would not expect to see significant bulk motions in the radial or vertical directions. However, in the left column of Figure 2, the majority of the bins in both panels at $\theta<0^{\circ}$ show non-zero velocities. Furthermore, bins above the plane nearly all have large positive (outward) $\left\langle V_{R}\right\rangle$, while stars in the symmetric region below the Galactic plane have either very small or slightly negative $\left(\left\langle V_{R}\right\rangle<0 \mathrm{~km} \mathrm{~s}^{-1}\right)$, inward-streaming motions. If the stars sampled disk-like rotational motion, we also should not see a change in $V_{R}$ across the Galactic anticenter. However, in this sample, the third Galactic quadrant $\left(\theta>0^{\circ}\right)$ has velocities that are nearly zero in most bins, as expected. Then, near $\theta=0^{\circ}$, the velocities change to become significantly non-zero in the second Galactic quadrant.

The lower left panel of Figure 2 shows that for the same stars at $\theta<0^{\circ}$ that have opposite $\left\langle V_{R}\right\rangle$ velocities above and below the plane, the $\left\langle V_{Z}\right\rangle$ components also differ. Specifically, the stars above $Z \sim 0.4 \mathrm{kpc}$ at $\theta<0^{\circ}$ nearly all have downward $\left(\left\langle V_{Z}\right\rangle<0 \mathrm{~km} \mathrm{~s}^{-1}\right)$ motions, while nearly all of the bins below the Galactic plane have $\left\langle V_{Z}\right\rangle>0 \mathrm{~km} \mathrm{~s}^{-1}$. 

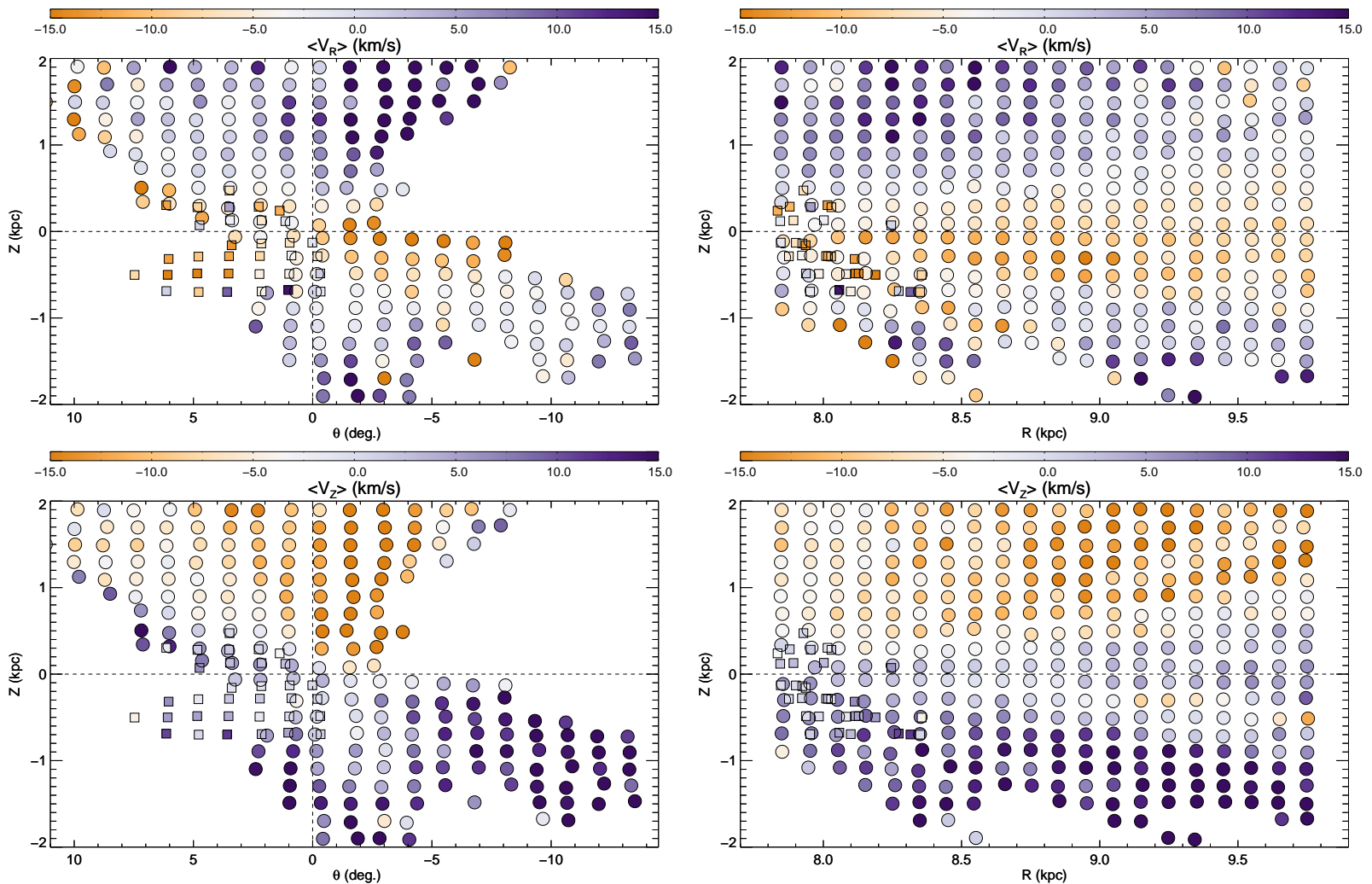

Fig. 2.- Radial ( $V_{R}$, upper panels) and vertical ( $V_{Z}$, lower panels) components of the Galactocentric velocities of stars between $7.8<R_{\mathrm{GC}}<9.8 \mathrm{kpc}$ as a function of positions in $Z$ and $\theta$ (left column) and $Z, R$ (right column). Circles denote LAMOST data, and small squares are derived from RAVE velocities. Each colored point represents the mean value of all stars within a bin $200 \mathrm{pc}$ wide in $R$ and $Z$, and $1.3^{\circ}$ in $\theta$. All bins contain at least 50 stars, and some contain many thousands of stars. The dots are centered at the mean position of the stars within each subsample, and color encodes the mean $V_{R}$ or $V_{Z}$ according to the scale given by the color bar at the top. Apparent radial features in the right panels are artifacts consistent with $\sim 20-30 \%$ errors in the distances.

The right column of Figure 2 can be compared to contour plots of RAVE red clump giants seen in Figure 11 of Williams et al. (2013, note that these authors adopted $R_{0}=8 \mathrm{kpc}$ rather than the value of $7.8 \mathrm{kpc}$ we used). Indeed, for the region between $8<R_{\mathrm{GC}}<10 \mathrm{kpc}$, the RAVE data exhibit similar asymmetries to those we see in LAMOST; namely, $\left\langle V_{R}\right\rangle>0 \mathrm{~km} \mathrm{~s}^{-1}$ above the midplane, and negative below the plane, while $\left\langle V_{Z}\right\rangle$ shows stars moving downward in regions above the plane and upward for $Z<0 \mathrm{kpc}$. For regions outside the solar radius, the RAVE data of Williams et al. (2013) are nearly all at $0^{\circ} \lesssim \theta \lesssim 14.4^{\circ}$ and longitudes $l \gtrsim 225^{\circ}$. It is remarkable, then, that the same asymmetrical velocity features we see predominantly near the Galactic anticenter and in the second quadrant are also present in this RAVE sample. 


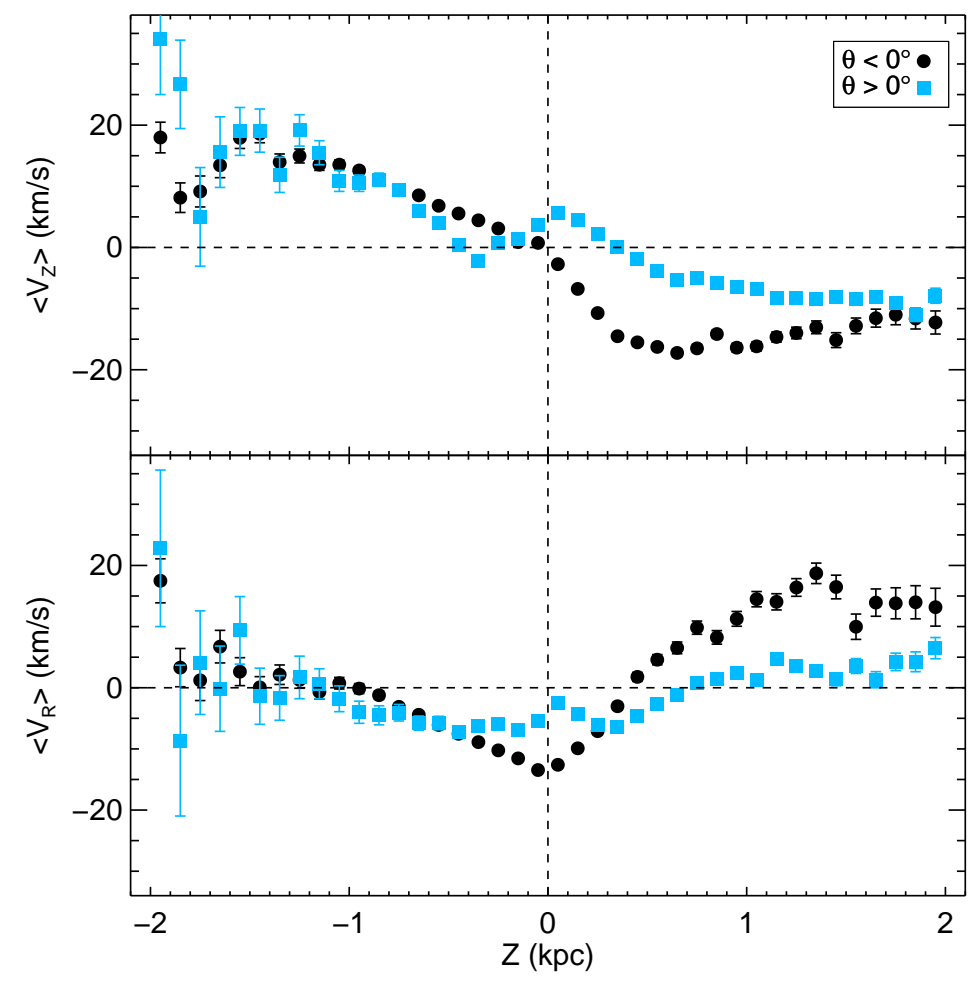

Fig. 3.- Mean $V_{Z}$ and $V_{R}$ velocities of stars as a function of $Z$ in $0.1 \mathrm{kpc}$ wide bins. Stars are separated into $\theta>0^{\circ}$ (blue filled squares) and $\theta<0^{\circ}$ (black filled circles) samples to examine differences between the second and third Galactic quadrants. Error bars on each point represent the standard error on the mean; for points that average hundreds or thousands of stars, the error bars are smaller than the point size.

To explore the azimuthal dependence (specifically, the difference between the second and third Galactic quadrants) of the vertical velocity asymmetries, we bin the data in a different way. Beginning with the entire data set of $\sim 400,000$ stars, we separate them at the Galactic $X$-axis to create $\theta>0^{\circ}$ and $\theta<0^{\circ}$ samples. These subsets contain 212,264 and 178,632 stars, respectively. For each subset, we group the data into $0.1 \mathrm{kpc}$ bins in $Z$ and calculate mean velocities $\left\langle V_{R}\right\rangle$ and $\left\langle V_{Z}\right\rangle$ for each bin. These are plotted in Figure 3 as a function of $Z$. There are significant differences between the two samples, especially above the Galactic plane, but the overall trends are similar $\left\langle V_{Z}\right\rangle$ is positive below the plane and negative at $Z>0$. In $\left\langle V_{R}\right\rangle$, the $\theta>0^{\circ}$ sample has smaller variations than the $\theta<0^{\circ}$ subset, but shows a similar trend toward positive $\left\langle V_{R}\right\rangle$ above the plane. There also appear to be small-scale wiggles (e.g., within $\pm 0.5 \mathrm{kpc}$ of the plane in $\left\langle V_{Z}\right\rangle$ ) in the mean velocity that may be akin to the coherent wavelike motions seen by Widrow et al. (2012) in SDSS data. 
We explored whether the unexpected motions in $\left\langle V_{R}\right\rangle$ and $\left\langle V_{Z}\right\rangle$ could be due to systematic errors in the kinematics and/or distances. Measured line-of-sight velocities (which have at most only

small systematic shifts; Luo et al. 2012) at the Galactic anticenter directly reflect the Galactocentric $V_{R}$ motions, but away from the anticenter the line-of-sight velocities give only a fraction of $V_{R}$. Because much of the velocity asymmetry we see is at $|Z| \gtrsim 1 \mathrm{kpc}$, it may be sensitive to systematic shifts in the proper motions. Artificially shifting $\mu_{\alpha} \cos \delta$ primarily changes $V_{R}$ in trends parallel to the Galactic plane (i.e., the magnitude of the change induced by shifts in $\mu_{\alpha} \cos \delta$ is roughly constant at a given $|Z|$ ), while $\mu_{\delta}$ systematically shifts regions at $|Z| \gtrsim 0.5 \mathrm{kpc}$ in the same direction and by roughly the same amount above and below the plane. It is unlikely that systematic proper motion errors can account for the asymmetries that we see both across the plane and across $\theta=0^{\circ}$. The $\left\langle V_{Z}\right\rangle$ component is primarily sensitive to the line-of-sight velocity above $|Z| \gtrsim 1 \mathrm{kpc}$. The vertical asymmetry in $\left\langle V_{Z}\right\rangle$ is thus robustly determined by spectroscopic measurements alone, though somewhat less secure at low latitudes, where the $Z$-component of Galactic velocities becomes sensitive to proper motion. The effect of shifting the distances mostly shifts the mean velocities in each bin by roughly the same amount, leaving the differences that make up the asymmetry roughly the same. Finally, by repeating the analysis on a subset of LAMOST spectra that have stellar parameters available, we verified that contamination by giant stars has little effect on our results.

\section{Conclusion}

We have shown an asymmetry in Galactocentric radial and vertical velocities across the midplane near the Galactic anticenter. F-type stars between $7.8<R_{\mathrm{GC}}<9.8 \mathrm{kpc}$ (i.e., within $2 \mathrm{kpc}$ outside the Solar radius) and $|Z|<2 \mathrm{kpc}$ are, on average, moving radially outward and downward toward the plane for positions above the midplane, and radially inward and upward toward the midplane for $Z<0$. This persists over a large region in azimuth that is covered by LEGUE. The maximum velocity difference between regions above/below the plane is $\sim 35 \mathrm{~km} \mathrm{~s}^{-1}$ in $\left\langle V_{Z}\right\rangle$, and $\sim 20 \mathrm{~km} \mathrm{~s}^{-1}$ in $\left\langle V_{R}\right\rangle$. The asymmetry seems to weaken with azimuthal angle $\theta$ toward the third Galactic quadrant.

A comparison with results from RAVE (Williams et al. 2013) shows that the same behavior is seen in the outer regions $\left(R_{\mathrm{GC}} \gtrsim 8.5 \mathrm{kpc}\right)$ probed by their data - stars above the plane have net downward motions directed radially outward, while those below the plane are on average moving upward and in toward the Galactic center. At first glance, this would seem to be contradicted by the SDSS results of Widrow et al. (2012), in which the stars in their Figure 4 at $Z<0$ kpc have $\left\langle V_{Z}\right\rangle<0 \mathrm{~km} \mathrm{~s}^{-1}$. These SDSS stars are located in the second Galactic quadrant (i.e., at $\theta<0^{\circ}$ ), where we find the strongest vertical asymmetry. Likewise, a similar negative $\left\langle V_{Z}\right\rangle$ is seen for stars below the plane from SDSS Stripe 82 (also mostly at $\left.\theta<0^{\circ}\right)$ in the analysis by Smith et al. (2012, see their Figure 6), coupled with a positive $\left\langle V_{R}\right\rangle$ for these same stars. However, we argue that this is consistent with our findings. Both Widrow et al. (2012) and Smith et al. (2012) restricted their stellar samples to $7<R_{\mathrm{GC}}<9 \mathrm{kpc}$ (with the Sun placed at $R_{0}=8 \mathrm{kpc}$ ) and used subsets of 
SDSS stars that probe different regions of the disk than our data. The RAVE data in Figure 11 of Williams et al. (2013) span $6.5 \lesssim R_{\mathrm{GC}} \lesssim 10.0 \mathrm{kpc}$, and within this figure one can see that $\left\langle V_{Z}\right\rangle$ and $\left\langle V_{R}\right\rangle$ change significantly with position. Thus it is not surprising that the different (and smaller) volumes probed by the SDSS studies compared to our LAMOST sample should show different velocity trends than we find.

This velocity asymmetry is likely the result of a vertical disturbance of the disk by an external perturber (Widrow et al. 2012), such as the Sagittarius dwarf galaxy (as suggested by Purcell et al. 2011; Gómez et al. 2013). The wavelike structure found in SDSS by Gómez et al. (2012), Smith et al. (2012), and Widrow et al. (2012) is likely related to the substructure found in different volumes by Williams et al. (2013) in RAVE data, and by our study of LAMOST data at slightly larger radii. Schönrich (2012) showed that systematic errors in the line-of-sight velocities from SDSS could account for the north/south velocity asymmetry shown by Widrow et al. (2012). However, the magnitude of the vertical velocity difference shown by LAMOST velocities in our study suggests that such a systematic difference cannot be solely responsible for the observed substructure. Finally, we note that there is an overdensity seen in SDSS at $R_{\mathrm{GC}} \sim 9.5 \mathrm{kpc}$, $Z \sim 0.6 \mathrm{kpc}$, and $Y>0$ by Jurić et al. (2008, see their Figures 26 and 27), which may correspond to the regions in our study that show net outward and downward motions.

All of these pieces of evidence are merely suggestive; much larger data sets and more thorough analysis will be required to map the coherent velocity features in three dimensions. With more extensive mapping of kinematics in the extended solar neighborhood enabled by large spectroscopic surveys such as RAVE and LAMOST, and ultimately by the Gaia mission, further constraints can be placed on the merging and interaction history of the Milky Way.

We thank Brian Yanny for sharing the spectroscopic data from the Widrow et al. (2012) study, and Mary Williams and Matthias Steinmetz for kindly sharing the RAVE data from Williams et al. (2013). We are grateful to the anonymous referee for thoughtful and helpful comments. This work was supported by NSF grants AST 09-37523 and NSFC grant 10973015. Undergraduate research support was provided by the NASA/NY Space Grant fellowship and NSF grants AST 10-09670 and DMR 0850934. Guoshoujing Telescope (the Large Sky Area Multi-Object Fiber Spectroscopic Telescope, LAMOST) is a National Major Scientific Project built by the Chinese Academy of Sciences. Funding for the project has been provided by the National Development and Reform Commission. LAMOST is operated and managed by the National Astronomical Observatories, Chinese Academy of Sciences.

\section{REFERENCES}

Antoja, T., Valenzuela, O., Pichardo, B., et al. 2009, ApJ, 700, L78

Cardelli, J. A., Clayton, G. C., \& Mathis, J. S. 1989, ApJ, 345, 245 
Carlin, J. L., Lépine, S., Newberg, H. J., et al. 2012, Research in Astronomy and Astrophysics, 12, 755

Chen, L., Hou, J.-L., Yu, J.-C., et al. 2012, Research in Astronomy and Astrophysics, 12, 805

Cui, X.-Q., Zhao, Y.-H., Chu, Y.-Q., et al. 2012, Research in Astronomy and Astrophysics, 12, 1197

Deng, L.-C., Newberg, H. J., Liu, C. et al. 2012, Research in Astronomy and Astrophysics, 12, 735

Fux, R. 2001, A\&A, 373, 511

Gómez, F. A., Minchev, I., O’Shea, B. W., et al. 2013, MNRAS, 429, 159

Gómez, F. A., Minchev, I., O’Shea, B. W., et al. 2012, MNRAS, 423, 3727

Gontcharov, G. A. 2006, Astronomy Letters, 32, 759

Haywood, M. 2008, MNRAS, 388, 1175

Jurić, M., Ivezić, Z., Brooks, A., et al. 2008, ApJ, 673, 864

Liu, X.-W., Yuan, H.-B., Huo, Z.-Y., et al. 2013, arXiv:1306.5376

Luo, A.-L., Zhang, H.-T., Zhao, Y.-H., et al. 2012, Research in Astronomy and Astrophysics, 12, 1243

McMillan, P. J., \& Binney, J. J. 2010, MNRAS, 402, 934

Minchev, I., Boily, C., Siebert, A., \& Bienayme, O. 2010, MNRAS, 407, 2122

Minchev, I., \& Famaey, B. 2010, ApJ, 722, 112

Minchev, I., Quillen, A. C., Williams, M., et al. 2009, MNRAS, 396, L56

Perryman, M. A. C., Lindegren, L., Kovalevsky, J., et al. 1997, A\&A, 323, L49

Purcell, C. W., Bullock, J. S., Tollerud, E. J., Rocha, M., \& Chakrabarti, S. 2011, Nature, 477, 301

Quillen, A. C., Dougherty, J., Bagley, M. B., Minchev, I., \& Comparetta, J. 2011, MNRAS, 417, 762

Quillen, A. C., \& Minchev, I. 2005, AJ, 130, 576

Roeser, S., Demleitner, M., \& Schilbach, E. 2010, AJ, 139, 2440

Schlegel, D. J., Finkbeiner, D. P., \& Davis, M. 1998, ApJ, 500, 525

Schönrich, R. 2012, MNRAS, 427, 274 
Schönrich, R., Binney, J., \& Dehnen, W. 2010, MNRAS, 403, 1829

Sellwood, J. A., \& Binney, J. J. 2002, MNRAS, 336, 785

Siebert, A., Williams, M., Siviero, A., et al. 2011, AJ, 141, 187

Skrutskie, M. F., Cutri, R. M., Stiening, R., et al. 2006, AJ, 131, 1163

Smith, M. C., Whiteoak, S. H., \& Evans, N. W. 2012, ApJ, 746, 181

Steinmetz, M., Zwitter, T., Siebert, A., et al. 2006, AJ, 132, 1645

Véron-Cetty, M.-P., \& Véron, P. 2010, A\&A, 518, A10

Widrow, L. M., Gardner, S., Yanny, B., Dodelson, S., \& Chen, H.-Y. 2012, ApJ, 750, L41

Williams, M. E. K., Steinmetz, M., Binney, J., et al. 2013, ArXiv e-prints:1302.2468

Wu, Z.-Y., Ma, J., \& Zhou, X. 2011, PASP, 123, 1313

Yang, F., Carlin, J. L., Liu, C., et al. 2012, Research in Astronomy and Astrophysics, 12, 781

Yanny, B., Rockosi, C., Newberg, H. J., et al. 2009, AJ, 137, 4377

Zhang, Y.-Y., Carlin, J. L., Yang, F., et al. 2012, Research in Astronomy and Astrophysics, 12, 792

Zhao, G., Zhao, Y.-H., Chu, Y.-Q., Jing, Y.-P., \& Deng, L.-C. 2012, Research in Astronomy and Astrophysics, 12, 723

Zwitter, T., Matijevič, G., Breddels, M. A., et al. 2010, A\&A, 522, A54 